\title{
Computer-assisted multi-needle surgical planning for large liver tumor radiofrequency ablation
}

\author{
Li Mengjiao', Qu Zhiyi ${ }^{*}$ \\ ${ }^{1,2}$ School of Information Science \& Engineering Lanzhou University \\ Lanzhou,China

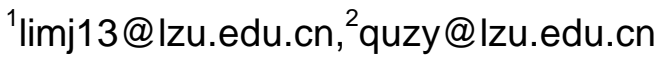

Keywords: multi-needle surgical planning, large tumor therapy, radiofrequency ablation, $3 \mathrm{~d}$ segmentation

Abstract. Multi-needle surgical planning is a quite complex and difficult job for doctors since the desired result is not very intuitive and has many constrains. The aim of this paper is let the computer automatically compute the operation scheme of the large liver tumor radiofrequency ablation, including puncture needles' number and their respective path. To solve the problem we proposed a semiautomatic approach based on 3D level set to segment liver tumor from CT volume. And based on the construction of the segmentation results, we proposed an algorithm to get an expected operation scheme. We develop a system implement the algorithm in this paper. It can help doctor to complete the surgical planning part almost automatically. The approach we proposed also can be used in other similar system.

\section{Introduction}

Primary liver tumors and liver metastases are of high clinical relevance. As a technique used to treat cancer, radiofrequency ablation is welcomed by doctors and patients since its minimally invasive, little pain and high security features[1]. RFA can locally destroy tumors using a percutaneous approach. And to apply this technology to the treatment of hepatocellular carcinoma and metastasis liver carcinoma, it also has achieved good clinical effect[2].

In most radiofrequency ablation surgery procedures, doctors insert a puncture needle into the tumor, and then RFA process starts denaturizing the cells in ablation zone. For the small tumor, which only need once ablation puncture, doctors relying on experience can quickly find a desired puncture path, in image guided. But when the tumor is large, in order to prevent recurrence the tumor must be covered entirely by the ablation area. Use a single puncture needle to puncture several times is a viable strategy[3]. At this time of puncture path is not very intuitive, the doctor is very difficult to plan out the desired path. It is very meaningful in using computer to plan surgery path automatically, in such a situation. It also would be a very challenging job to make multiple needles puncture by the doctors, even if the problem of path planning can be solved. Doctors need to make sure that each needle puncture should be fairly accurate, while that in the case of multiple needles puncture is not easy to do. Using robots to assist doctors to puncture, can significantly reduce the burden of doctors and improve the quality of surgery because of the robot's characteristics of good stability and high precision.

To let the computer can automatically get the operation scheme according to our requirements, we proposed an approach to solve the problem. Our method based on CT image guided is effective and convergent. And in order to image preprocessing, we also proposed an method based on $3 \mathrm{~d}$ level set to segment the tumor from the $\mathrm{Ct}$ volume. We develop a system based on above algorithms that can output the desired operation scheme.

\section{Related work}

In the current medical robot system, virtual reality, 3d reconstruction and collision detection technology are often used in surgery planning. Petehans et al. [4] has developed a three-dimensional navigation platform for liver tumors. Japanese Renishaw company have developed a preoperative 
planning system named Neuro Mate[5], it can simulate the process of piercing after it get the 3d reconstruction of lesion group and the puncture route. But these systems only adapt to the simple single needle puncture, and cannot help doctors to reduce the financial burden on the actual operation. Laurent et al. [6] proposed a method to choice the puncture target point and it achieve a desired result in a vitro experiment. Caroline et al. [7] have developed a simulation system for finding the puncture point in liver tumor and simulating the surgery. But these systems do not take the puncture point on skin into account, although it is very important in the actual operation. Liu [8,9] et al. puts forward a method to find the puncture point on skin. But they not yet implement their algorithm in a real environment.

\section{Methods}

Goals of multi-needle surgical planning. In traditional surgery process, after the patient is under local anesthesia, the doctor would choose an appropriate puncture path according to the ultrasound guidance. And then the puncture needle be inserted into the predetermined position by doctors to destruct the tumor. For a small tumor, only a single puncture can destroy it. If the tumors had more than $3.5 \mathrm{~cm}$ diameter, they could be destroyed by two methods: firstly, use a cluster of needle instead of a single needle; secondly, use a single needle to puncture the tumor by several times [1]. In this paper, our solution is based on the strategy of the second.The work of this paper is mainly focused on the preoperative planning stage. The procedure of surgical planning is like this: firstly collect the CT images of the organization around the patient's liver before surgery. Then segment the main organization, such as bone, liver, vascular etc. and reconstruct them. Based on the $3 \mathrm{~d}$ model, get the solution by the planning algorithm.Because of the needle used in RFA has strong rigidity and it will not deformation, so its track is a straight line. The planning results can be expressed as a series of entities as follows: $\left\{\mathrm{P}_{\mathrm{A}}, \mathrm{P}_{\mathrm{B}}\right\} \in \mathrm{R}^{3} * \mathrm{R}^{3}$

$P_{A}$ represents the puncture position on the skin and $P_{B}$ is the corresponding position in the tumor. $\mathrm{P}_{\mathrm{A}} \mathrm{P}_{\mathrm{B}}$ is a expected path for a single RFA process. Several of them constituted the planning results.

Semiautomatic liver tumor segmentation with 3D level set. During our work, we found liver tumor segmentation is a very difficult task because of heterogeneous density and weak boundaries of the tumor. In addition, the tumor is usually surrounded by other structures that have similar intensity, which further complicates the processing. This paper proposes a semiautomatic approach to segment liver tumor from CT volume based on a 3D level set model. It only requires a few steps of user interaction to accomplish the segmentation. The pipeline of the proposed approach is shown in Fig.1. The user first specifies a three-dimensional ROI by indicating a rectangle that contains the tumor tissue in a 2D slice (Fig.1a) and choosing the slice range that includes the tumor. This will generate a cuboid that embraces the whole tumor according to the specified rectangle so as to remove irrelevant parts of the volume. After that, anisotropic diffusion filtering is applied to CT images to reduce the noise while preserving salient edges (Fig.1b). Then parameters of the level set formula will be computed based on the homogeneous region inside the tumor tissue or can be manually set by the user. Level set segmentation is performed on the filtered volume to produce the surface of the tumor (Fig.1c). Due to the heterogeneous intensity of tumor and the existence of neighboring tissues, there may be some disconnected segments in the final contour. In this regard, a few morphological operations are performed to remove the unwished segments and refine the segmentation result (Fig.1d).

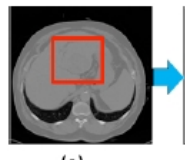

(a)

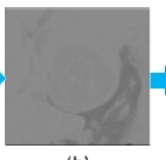

(b)

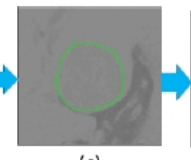

(c)

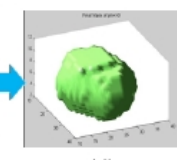

(d)

Figure 1: Pipeline of the proposed approach.

The anisotropic diffusion filter proposed by Perona and Malik [10] is employed to reduce the noise in CT images due to its capability to remove noise while preserving salient edges. This filter is defined as 


$$
U_{t}-\operatorname{div}(a(|\nabla u|) \nabla u)=0 \text { In } \Omega \text { for } \mathrm{t}>0 ; \partial_{v} u=0 \text { On } \partial \Omega \text { for } \mathrm{t}>0 ; u(0, \cdot)=u_{0} \operatorname{In} \Omega
$$

Where $u$ denotes an image, $\Omega$ is the image domain, $v$ represents the unit outer normal at the boundary, and $\mathbf{a}$ is a nonlinear diffusion coefficient.

Level set methods implicitly represent the evolution of the surface with a higher dimension function

$$
\partial \phi \dot{E} \partial t=-\nabla \phi \cdot \bar{v}(t)
$$

Where $\bar{v}(t)$ scribes the point-wise velocity of the surface. The velocity usually combines a data term and a regularization term as

$$
\frac{\partial \phi}{\partial t}=\left|p^{\varnothing}\right|\left[a D(\bar{x})+(1-a) \nabla \cdot \frac{\nabla \phi}{|\nabla \phi|}\right]
$$

We employ the level set model proposed in [11] to segment liver tumor by using the following data term $(I)=\varepsilon-|1-T|$, where $T$ and $\varepsilon$ denote the central intensity and deviation of pixel intensities in the target region that will be segmented, respectively. In this model, D forces the model toward desirable edges in the input data, $\nabla \cdot(\nabla \phi E \dot{E}|\partial \phi|)$ is the mean curvature of the surface that forces the surface to remain smooth, and $a \mathrm{U}[0,1]$ is a free parameter that controls the degree of smoothness. The up-wind differencing scheme [12] is used to discretize the level set equation. Curvature is computed using the difference of normal method from [13].

Puncture target spot in tumor planning. First of all, our method is based on a hypothesis that the effective ablation area would be seen as a sphere which center is the puncture needle tip in a single puncture process. And we set the sphere has a radius of y.In order to guarantee completely destroy the tumor, the tumor model would be expanded by its surrounding area, and the whole area be target areas. Depending on the shape of tumor, adjust the around area, make the total area become into a sphere. And we set the sphere has a radius of $\mathrm{x}$.

So the planning problem can be simplified as:Use less as far as possible sphere with the radius of $y$ to cover the $\mathrm{x}$ radius sphere. If $\mathrm{y}>\mathrm{x}$, just only need one $\mathrm{y}$ radius sphere obviously. But if $\mathrm{y}<\mathrm{x}$, the answer is not straightforward. For example, $\mathrm{y}=25$ and $\mathrm{x}=15$, what's the answer?

Even with the same number of puncture times, since the different arrangement, the final effect might be different.This paper proposes an algorithm to search for the best puncture points in the target area. The algorithm is based on voxel.The target area and its around tissue are stored into a $3 \mathrm{~d}$ matrix, each voxel represents $1 \mathrm{~mm} 3$ volume. We define the loss function as follow:

$$
L=\sum_{i=1}^{N} \sum_{t=1}^{M} \alpha v_{t}
$$

$\mathrm{N}$ is the number of puncture. $\mathrm{M}$ is the number of voxels impacted by a single RFA. $\mathrm{v}_{\mathrm{t}}$ Represents a certain voxel and $\alpha$ is the corresponding weight. Other cases, $\alpha$ is a positive number as a penalty term. Thus, the problem can be written as:

$$
\begin{gathered}
\text { Minimize: } L \\
\text { Subject to: }\left(\sum_{i=1}^{N} V_{i}\right) \cap T=T
\end{gathered}
$$

$\mathrm{T}$ represents the tumor's all voxels. Vi represents the whole voxels of the impacted area by one puncture needle.In the process of optimization, each single voxel is counted only once.Obviously as algorithm in the iteration, the center area of the tumor will be treated, and then the area of expansion will be covered, at last some healthy tissue may be impacted. We use Nelder-mead method [14] to solve the problem. It is a heuristic search algorithm, can greatly improve the computational efficiency.Finally, our result is a series of targets and has the following form $\left\{\mathrm{P}_{\mathrm{B} 1}, \mathrm{P}_{\mathrm{B} 2}, \mathrm{P}_{\mathrm{B} 3} \ldots \mathrm{P}_{\mathrm{Bn}}\right\}$.

Puncture spot on skin planning. In the last section we get the sequence of $\mathrm{P}_{B}$. For different $\mathrm{P}_{B}$ we require a corresponding $\mathrm{P}_{\mathrm{A}}$. Thus $\mathrm{P}_{\mathrm{A}} \mathrm{P}_{\mathrm{B}}$ can constitute a puncture path.

In the surgery, $\mathrm{P}_{\mathrm{A}}$ has the following constraints:

1) $P_{A}$ on the skin surface.

2) The path formed by $\mathrm{P}_{\mathrm{A}} \mathrm{P}_{\mathrm{B}}$ does not interference with obstacles such as bone, blood vessels and so on. 
3) The Angle between $\mathrm{P}_{A} \mathrm{P}_{B}$ and XOY (Figure 2.) plane should in a given range. Because puncture under different angles cause different pain degree felled by patient. And also, the scope of the robot's movement should be considered.

In this paper, we using the third order continuous differentiable super quadric surface ${ }^{[15]}$ to describe obstacles such as ribs and blood vessels. Their standard form in local coordinates as follow:

$$
f\left([x, y, z]^{T}\right)=\left(\frac{x}{a_{1}}\right)^{12}+\left(\frac{y}{a_{2}}\right)^{2}+\left(\frac{z}{a_{3}}\right)^{2}-1=0
$$

$[\mathrm{x}, \mathrm{y}, \mathrm{z}]^{\mathrm{T}}$ is the coordinates of the point on the surface in $\mathrm{R}^{3}$. $\mathrm{f}$ is convex function. Through homogeneous transformation, they will be placed to the right place.The abdominal skin fitting for the following forms of parametric equation:

$$
\mathrm{M}(\mathrm{u}, \mathrm{v})=[\mathrm{x}(\mathrm{u}, \mathrm{v}), \mathrm{y}(\mathrm{u}, \mathrm{v}), \mathrm{z}(\mathrm{u}, \mathrm{v})]^{\mathrm{T}}, \mathrm{u} \in\left[\mathrm{u}_{\min }, \mathrm{u}_{\max }\right], \mathrm{v} \in\left[\mathrm{v}_{\min }, \mathrm{v}_{\max }\right]
$$

The algorithm we used to search desired $\mathrm{P}_{\mathrm{A}}$ is as follows:

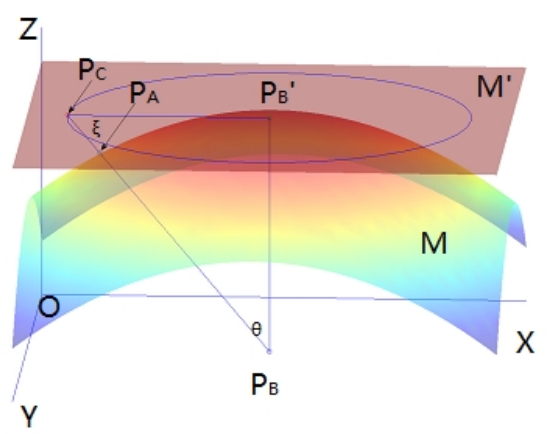

Figure 2: Find the skin puncture point diagram.

As shown in figure 2, fit a plane $M$ ' paralleled with XOY plane at $\mathrm{z}=\mathrm{h}$. $\mathrm{P}_{\mathrm{Bi}}$ projection on the $\mathrm{M}$ for $\mathrm{P}_{\mathrm{Bi}}$. Used $\mathrm{P}_{\mathrm{Bi}}$ as the center of circle, we make a $\mathrm{d}$ radius circle $\mathrm{A}$. $\mathrm{d}=\mathrm{h} * \tan \theta . \theta+\xi=\Pi / 2$. $\xi$ is the Angle between $\mathrm{P}_{\mathrm{A}} \mathrm{P}_{\mathrm{B}}$ and XOY plane.After input $\xi$, we can get $\theta$. Set $\mathrm{P}_{\mathrm{ci}}$ as the starting point and update $\mathrm{P}_{\text {ci }}$ on the circumference of circle $\mathrm{A}$ in step $\beta$, until we find a $\mathrm{P}_{\text {cit }}$ meet $\mathrm{P}_{\mathrm{Bi}} \mathrm{P}_{\text {cit }}$ and obstacle's distance is greater than the $\gamma$. Then the intersection $\mathrm{P}_{\mathrm{Ai}}$ of $\mathrm{M}$ and $\mathrm{P}_{\mathrm{Bi}} \mathrm{P}_{\mathrm{Cit}}$ is the solution. Which the value of $\beta$ and $\gamma$ is set according to the specific circumstances. Adopting literature, we use the interior point method ${ }^{[16]}$ to calculate the distance between $\mathrm{P}_{\mathrm{Bi}} \mathrm{P}_{\mathrm{Cit}}$ with the obstacle.

\section{Results and Simulations}

we developed a system which functions integrates the ct-scan, $3 \mathrm{~d}$ reconstruction and surgery planning etc. Figure 3 and figure 4 shows some features of the system.

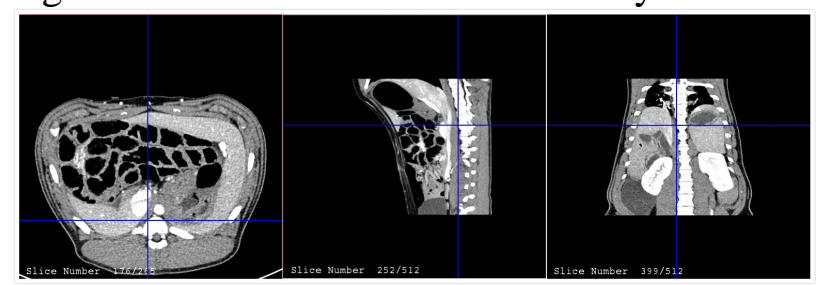

Figure 3: Ct scan function of the system. Upper left, upper right, lower left in turn is transverse view, coronal view and sagittal view.

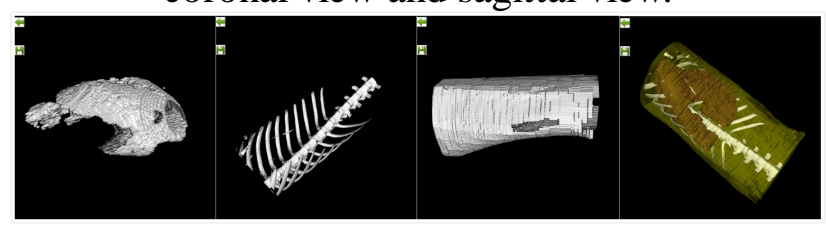

Figure 4: 3D reconstruction function of the system.

The rendering part of the system based on VTK library [17]. It runs on the workstation that installed Windows 7 system and use Intel core i3 processor and has 4 GB memory. We collected the CT images of the parts of pig's liver as the original data to verify the algorithm proposed in this paper. The dimension of each CT image is $512 * 512$, and the slice thickness is $1 \mathrm{~mm}$. Totally there are 256 slices. 
Fig. 5 shows the effect of different iteration number with other parameters the same. To obtain a connected and smooth surface of the tumor, contours resulted from level set segmentation are refined by a few morphological operations. Holes are first filled and then the unwished segments are removed to get the final result.

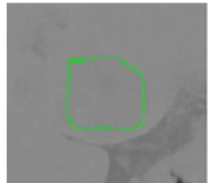

(a)

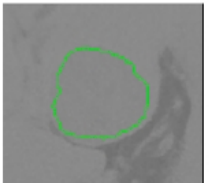

(b)

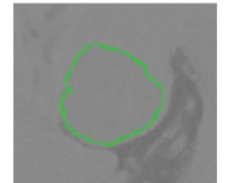

(c)

Figure 5: Segmented contours with different iteration number: (a) ite $=69$, (b) ite $=219$, (c) ite $=419$.

Fig. 6 presents the segmentation result of the tumor with a small cube as initial surface. In this example, the parameters are set as follows: ite $=399, \mathrm{~T}=1088, \varepsilon=15$.
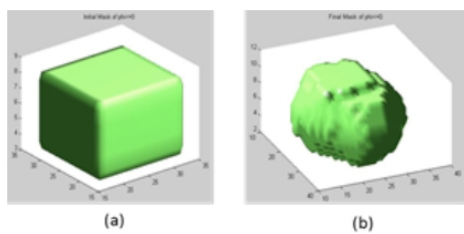

Figure 6: Initial surface (a) and final surface (b).

Assuming the largest diameter of the tumor area is less than $50 \mathrm{~mm}$, then the radiofrequency ablation target area is a spherical radius of $25 \mathrm{~mm}$ with the center of tumor as its center. Choose the puncture needle with $15 \mathrm{~mm}$ radius of ablation to surgery. Figure 7 shows the planning results. First and second presents the situation that after the fifth puncture. The third presents the situation after twentieth puncture. The fourth displays the position of the twenty needle placement.It will be altogether 20 puncture points in the tumor, and the coverage of the ablation target area is $100 \%$. They will affect $7878 \mathrm{~mm} 3$ normal tissue at the same time.

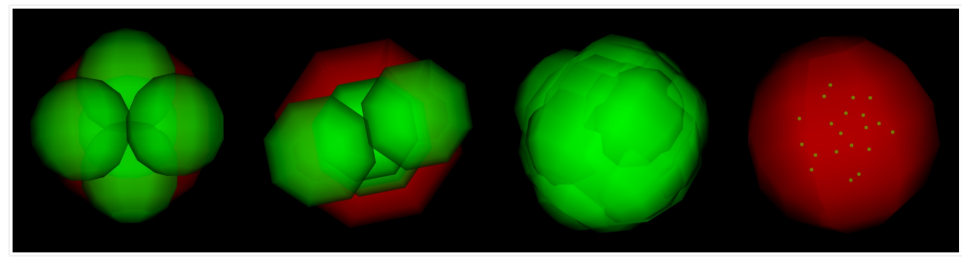

Figure 7: The result of needle placement in tumor.

Figure 8 shows the planning results for a real tumor. Left is a real tumor. Right is the results.

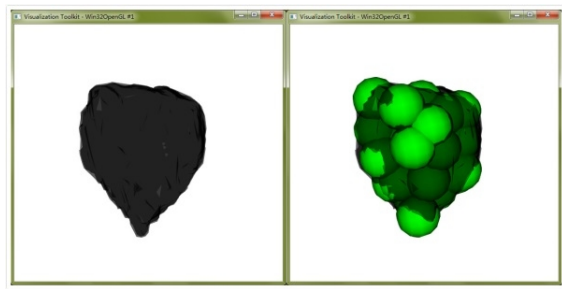

Figure 8: The planning results for a real tumor.

After got the coordinates of the puncture target, we set $\theta=30, \beta=1$ to find the location of the corresponding skin puncture point as the algorithm we proposed.

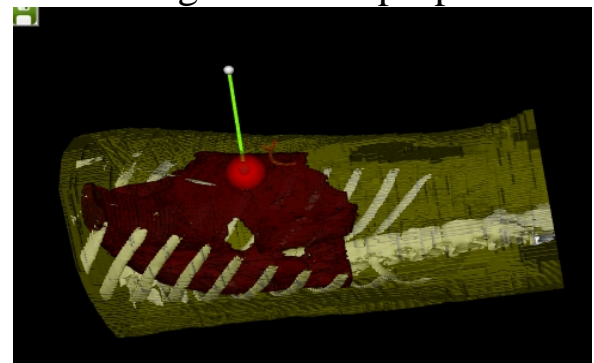

Figure 9: One corresponding puncture path of two puncture target point in tumor. 
Figure 9 shows the corresponding path of one puncture target points.As you can see, the planning of the path meets the needs of the surgery. The set of all puncture target points' corresponding path is our planning results.

\section{Conclusion}

In this paper, an approach segment the tumor from CT image and an algorithm that get the desired puncture path in RFA are proposed. Our algorithms are robust that parameters can be changed according to the actual situation. And our algorithm can obtain an good effect with little iterations.

Based on the algorithm, we developed a system that integrates the ct-scan, semi-automatic segmentation, $3 \mathrm{~d}$ reconstruction and surgery planning, and other functions. The final planning result can be export and used for the piercing robot control system.Our subsequent work will focus on the factors such as soft tissue deformation, breathing exercises that may affect accuracy of operation.

\section{Reference}

[1] Liang P et al. Ultrasound-guided microwave coagulation therapy for liver cancer[M]. Beijing: People's Military Medical Press, 2003.

[2] Liang P. Clinical application of ultrasound (US)-guided percutaneous microwave coagulation therapy for liver cancer[J]. CHINESE JOURNAL OF ONCOLOGY, 1997, 19(6):448-450.

[3] $\mathrm{Xu}$ J. The robotic system of microwave coagulation therapy for liver cancer based on ultrasound navigation[J]. ROBOT, 2007, 29(5).

[4] Peterhans M, Berg A V, Dagon B, et al. A navigation system for open liver surgery: design, workflow and first clinical applications[J]. International Journal of Medical Robotics and Computer Assisted Surgery, 2011, 7(1): 7-16.

[5] Renishaw. Surgical planning software[EB/OL]. [2011-06-18]. http://www.renishaw.com/en/surgical-planning-software--8244.

[6] Mundeleer L et al. Computer-assisted needle positioning for liver tumor radiofrequency ablation(RFA)[J]. THE INTERNATIONAL JOURNAL OF MEDICAL ROBOTICS AND COMPUTER ASSISTED SURGERY, 2009(5):458-464.

[7] Caroline V. et al. Towards realistic radiofrequency ablation of hepatic tumors 3D simulation and planning[C]. //Medical Imaging 2004:Visualization,Image-Guided Procedures, SPIE, 2004.

[8] Liu S. Analytic Description of Medical Robot Needles' Collision-free Reachable Workspace Boundary[J]. ROBOT, 2012, 34(1).

[9] Liu S. Multi-needle surgical planning for medical robot in large tumor therapy[J]. ROBOT, 2013, 35(6).

[10]P. Perona and J. Malik, Scale-Space and Edge Detection Using Anisotropic Diffusion, IEEE Transactions on Pattern Analysis and Machine Intelligence, Vol. 12, No. 7, pp. 629-639, 1990.

[11]A. E. Lefohn, J. E. Cates, and R. T. Whitaker Interactive, GPU-Based Level Sets for 3D Segmentation, MICCAI, pp. 564-572, 2003.

[12]S. Osher and R.P. Fedkiw. Level set methods and dynamic implicit surfaces. Springer, 2003.

[13]Lefohn, Aaron E., et al. A streaming narrow-band algorithm: interactive computation and visualization of level sets. ACM SIGGRAPH 2005 Courses. ACM,2005.

[14] W.H. Press, S. A. Teukolsky, W. T. Vetterling, and B. P. Flannery, Numerical Recipes in C++: The Art of scientific Computing (second Edition), Cambridge University Press, 2002.

[15]Hanson A J. Hyperquadrics: Smoothly deformable shapes with convex polyhedral bounds[J]. Computer Vision, Graphics, and Image Processing, 1988, 44(2): 191-210.

[16]Chakraborty N, Peng J, Akella S, et al. Proximity queries between convex objects: An interior point approach for implicit surfaces[J]. IEEE Transactions on Robotics, 2008, 24(1): 211-220.

[17] Schroeder, William J. Visualizing with VTK: A tutorial[C]. //IEEE Computer Graphics and Applications, 2000. 